\title{
Aches and pains in primary care: stay positive but critical
}

The normal state of humankind is to experience some pain some of the time. The dominant causes of chronic pain globally are musculoskeletal conditions such as back pain and osteoarthritis. They tend not to kill people, and mild forms are regarded as an inevitable part of life. GPs, some would say, should concern themselves with the assessment and investigation of pain as a pointer to serious diseases such as cancer or coronary atheroma.

Yet the very frequency of aches and pains also signals their importance. Chronic musculoskeletal pain restricts physical activity and participation in social and domestic life. And so common are musculoskeletal conditions that they dominate disability in developed countries and many developing countries as well; ${ }^{1}$ back pain is second only to mental illness as a reason for long-term work loss; and osteoarthritis is likely to overwhelm other chronic diseases as a cause of years lived with restricted activity in the future.

This is hardly news for GPs. One-quarter of primary care consultations concern musculoskeletal aches and pains, ${ }^{2}$ a frequency only surpassed in UK general practice by consultations for respiratory problems. $^{3}$

There are occasional success stories for biomedical approaches to these conditions; for example, when uncommon but important serious underlying causes, such as inflammation, are diagnosed and treated, or when a joint is replaced. But there are problems in applying traditional medical models to such common conditions. These models focus on the search for a diagnosis when what is needed for many patients in pain are ways to help them maintain function in their daily lives. Chronic pain management dominated by analgesic medication fails to address the role of activity and psychological and social factors in maintaining daily function. And negative perceptions and frustrations about the importance and the possibility of doing something for chronic aches and pains are common among patients, clinicians, and researchers. Two papers in this month's Journal ${ }^{4,5}$ remind us of the continuing need to combine critical reflection on whether the natural course of pain can be improved by biomedical advances, such as diagnostic technology, with positive active approaches to management.

The study of knee injuries from the Netherlands illustrates the type of careful clinical epidemiological study still needed in a world focused on randomised controlled trials. ${ }^{4}$ It confirms that average outcome for acute pain precipitated by events such as sports injury is good, and that the outcome of knee pain is largely independent of baseline MRI diagnosis. As the authors point out, this is only half the story since they did not follow long-term outcomes (meniscal tears are related to future osteoarthritis ${ }^{6}$ but it is unclear if repair reduces that risk $^{7}$ ). Outcomes might have been different if MRI-linked treatment had been provided straightaway (the DAMASK trial suggested small advantages in pain relief with this strategy. $\left.{ }^{8}\right)$ However evidence from other conditions suggests the disadvantages of overinvestigation. A randomised controlled trial in back pain consulters found MRI findings increased the numbers of diagnoses made and interventions delivered, with no improvement in pain and disability compared with the group not having MRI. ${ }^{9}$ Such intervention studies can investigate the effectiveness of treatment linked to diagnosis, but observational studies, such as that by Wagemakers et $a l,{ }^{4}$ create the critical question: 'Given you make a diagnosis on the basis of this new investigation or technology, is the prognosis any different in the subgroups identified?'

The critical perspective provided by prognosis studies extends to the traditional separation of chronic musculoskeletal pain syndromes based on the site of the body affected (shoulder versus back for example) and the underlying assumption that these are distinctive diseases with their own aetiology. Generic factors, such as pain severity or psychological and social characteristics, that are similar across the individual regional syndromes, appear more important than diagnostic features for predicting prognosis in individual patients with chronic pain. ${ }^{10}$

Analgesic prescriptions are the most frequent treatment for chronic aches and pains in primary care, with two-thirds of persons who consult with musculoskeletal conditions receiving one. ${ }^{2}$ They are clearly important for acute, traumatic, or end-oflife pain. But there is little evidence that analgesics reduce chronic pain and its attendant disability beyond 6 months. This applies to opioids as well as to weaker analgesics, ${ }^{11}$ and yet the use of the former for chronic musculoskeletal pain is rising in the US and Europe with no evidence about long-term safety. This reflects the concerns about taking long-term medication for osteoarthritis expressed by the focus groups in Williams et al's paper on page $113 .{ }^{5}$

Nonpharmacological interventions also need to be shown as safe to use, but are intuitively more attractive than medications as long-term options for chronic pain management - especially if they engage and support patients in self-management or address shifts in behaviour and attitudes about pain that encourage activity (such as exercise or work). Williams and colleagues describe the development of a booklet for sufferers from hip and knee osteoarthritis to help and support activity, using similar principles to a widely used booklet for back pain. ${ }^{5}$ From one perspective, it is simply appropriate practice for primary care to provide such information regardless of specific effects on pain or disability. But it is important also not to be complacent about the extent of these effects, which in isolation may be limited. In osteoarthritis, provision of support and advice from expert patients improved new patients' confidence about what they could achieve but with no clear evidence of changes in pain and daily activity. ${ }^{12}$ Back pain has appeared more responsive, and 
Buchbinder and colleagues have shown that media campaigns to support the provision of information and advice can affect population behaviours such as sickness absence rates. ${ }^{13}$ Attempts to replicate this elsewhere than Australia, however, suggest that, while public and healthcare professional perceptions change, behavioural or clinical outcomes, such as lower rates of pain or work loss, do not necessarily follow.

However, it is worth being positive. Patients are often told there is little to be done and that aches and pains are an inevitable consequence of ageing. In both back pain and joint pain, recent surveys of healthcare professionals have found extensive continuing use of language and approaches that discourage activity or early return to work. ${ }^{14,15}$ The capacity of such negative language to exacerbate persistence of aches and pains and restricted activity has yet to come under the research microscope, but it is interesting to read examples of the language in Williams et al's booklet emphasising the positive benefits of physical activity. ${ }^{5}$ The authors highlight also the problem of confused messages exercise can cause osteoarthritis (as it may do in elite Finnish marathon runners or professional footballers' knees overtreated with steroids) and yet is beneficial for osteoarthritis (as it is in moderation or particularly when advice is delivered by physiotherapists). Previous research on back pain has also highlighted the detrimental effect of inconsistency between printed and verbally delivered advice. ${ }^{16}$ The role of Williams' focus groups in pointing this out emphasises the need for public and patient involvement in the development of educational material.

The most compelling argument for 'stay positive but critical' rests on the sheer frequency of musculoskeletal pain in primary care. From a primary prevention perspective, public health targets such as injury prevention, obesity control, promotion of work as a positive contribution to health, and increased physical activity, would, on available evidence, improve population musculoskeletal health. In effect, because so much musculoskeletal pain is presented to primary care, health professionals in that setting have the opportunity to contribute to the public health endeavour through secondary prevention. If primary care professionals, such as GPs or physiotherapists, can steer patients to interventions that result in a modest reduction in pain, promote activity or participation despite the pain, or relieve the distress or catastrophising ideas about pain which can make pain worse or more disabling, then such effects regularly produced in many people could have a substantial impact on population levels of pain and disability and on the capacity to participate fully in social and domestic life. Diagnostic procedures or medications, however important for certain subgroups and individuals, alone cannot solve the problem, and might be costly and ineffective if applied to the total population of primary care patients with chronic pain.

\section{Peter Croft,}

Director, Arthritis Research Campaign, National Primary Care Centre, University, Keele.

\section{Provenance}

Commissioned; not peer reviewed.

\section{REFERENCES}

1. Demyttenaere K, Bruffaerts R, Lee S, et al. Mental disorders among persons with chronic back or neck pain: Results from the world mental health surveys. Pain 2007; 129: 332-342.

2. Hasselstrom J, Liu-Palmgren J, Rasjo-Wraak G. Prevalence of pain in general practice. Eur J Pain 2002; 6: $375-385$.

3. Arthritis Research Campaign National Primary Care Centre. Musculoskeletal Matters Bulletin No.1: what do general practioners see?

http://www.keele.ac.uk/research/pchs/pcmrc/dissemina tion/bulletin (accessed 14 Jan 2010).

4. Wagemakers HPA, Luijsterburg PAJ, Heintjes EM, et al. Outcome of knee injuries in general practice: 1-year follow-up. Br J Gen Pract 2010; DOI: 10.3399/bjgp10X483157.

5. Williams NH, Amoakwa E, Burton K, et al. The Hip and Knee Book: developing an active management booklet for hip and knee osteoarthritis. Br J Gen Pract 2010; DOI: 10.3399/bjgp10X483166.

6. Englund M, Guermazi A, Roemer FW, et al. Meniscal tear in knees without surgery and the development of radiographic osteoarthritis among middle-aged and elderly persons: The Multicenter Osteoarthritis Study. Arthritis Rheum 2009; 60: 831-839.

7. Englund M, Lohmander LS. Risk factors for symptomatic knee osteoarthritis fifteen to twenty-two years after meniscectomy. Arthritis Rheum 2004; 50: 2811-2819.

8. DAMASK (Direct Access to Magnetic Resonance Imaging: Assessment for Suspect Knees) Trial Team. Effectiveness of GP access to magnetic resonance imaging of the knee: a randomised trial. Br J Gen Pract 2008; DOI:10.3399/bjgp08X342651.

9. Jarvik JG, Hollingworth W, Martin B, et al. Rapid magnetic resonance imaging vs radiographs for patients with low back pain: a randomized controlled trial. JAMA 2003; 289: 2810-2818.

10. Mallen CD, Peat G, Thomas E, et al. Prognostic factors for musculoskeletal pain in primary care: a systematic

review. Br J Gen Pract 2007; 57: 655-661.

11. Ballantyne JC, Shin NS. Efficacy of opioids for chronic pain: a review of the evidence. Clin J Pain 2008; 24: 469-478.

12. Buszewicz M, Rait G, Griffin M, et al. Self management of arthritis in primary care: randomised controlled trial. BMJ 2006; 333: 879.

13. Buchbinder, R., Jolley, D., Wyatt, M. Population based intervention to change back pain beliefs and disability: three part evaluation. BMJ 2001; 322: 1516-1520.

14. Bishop A, Foster NE, Thomas E, Hay EM. How does the self-reported clinical management of patients with low back pain relate to the attitudes and beliefs of health care practitioners? A survey of UK general practitioners and physiotherapists. Pain 2008; 135: 187-195.

15. Holden MA, Nicholls EE, Young J, et al. UK-based physical therapists' attitudes and beliefs regarding exercise and knee osteoarthritis: findings from a mixed-methods study. Arthritis Rheum 2009; 61: 1511-1521.

16. Little $\mathrm{P}$, Roberts L, Blowers $\mathrm{H}$, et al. Should we give detailed advice and information booklets to patients with back pain? A randomized controlled factorial trial of a self-management booklet and doctor advice to take exercise for back pain. Spine 2001; 26: 2065-2072.

DOI: 10.3399/bjgp10X482301

ADDRESS FOR CORRESPONDENCE

\section{Peter Croft}

Director, Arthritis Research Campaign,

National Primary Care Centre, Keele

University, Keele ST5 5BG.

E-mail: p.r.croft@cphc.keele.ac.uk 